\title{
Functional Organization of a Neural Map in the Cricket Cercal Sensory System
}

\author{
Gwen A. Jacobs and Frédéric E. Theunissen \\ Department of Molecular and Cell Biology, University of California, Berkeley, California 94720
}

\begin{abstract}
Directionally selective mechanosensory afferents in the cricket cercal sensory system form a map of air current direction in the terminal abdominal ganglion. The global organization of this map was revealed by studying the anatomical relationships between an ensemble of sensory afferents that represented the entire range of receptor hair directional sensitivities on the sensory epithelium. The shapes and three-dimensional positions of the terminal arborizations of these cells were highly conserved across animals. Afferents with similar directional sensitivities arborized near each other within the map, and their terminal arborizations showed significant anatomical overlap. There was a clear global organization pattern of afferents within the map: they were organized into a spiral shape, with stimulus direction mapped continuously around the spiral. These results
\end{abstract}

demonstrate that this map is not formed via a direct point-topoint topographic projection from the sensory epithelium to the CNS. Rather, the continuous representation of air current direction is synthesized within the CNS via an anatomical reorganization of the afferent terminal arbors. The arbors are reorganized according to a functional property that is independent of the location of the mechanoreceptor in the epithelium.

The ensemble data were used to derive predictions of the patterns of steady-state excitation throughout the map for different directional stimuli. These images represent quantitative and testable predictions of functional characteristics of the entire neural map.

Key words: neural map; database; three-dimensional reconstruction; sensory system; insect; functional neuroanatomy
The functional characteristics of any neural map depend on two principal factors: the physiological properties of the neurons that comprise the map, and the global structural organization of their terminal arborizations within the CNS. The anatomical projections of the neurons in a neural map form the template on which their functional properties are represented and, hence, define the naturc of the functional interface to the next computational stage of the CNS. The goals of this study were to examine quantitatively how the projections of an array of receptor neurons are organized anatomically in the CNS to represent specific functional characteristics and to assess the effect of the anatomical and physiological characteristics of the receptor array on those representations.

The system we studied was the cricket cercal sensory system. This system is capable of detecting the direction and dynamics of behaviorally relevant air currents with great accuracy and precision (Shimozawa and Kanou, 1984a,b; Miller et al., 1991; Theunissen and Miller, 1991; Landolfa and Miller, 1995; Roddey and Jacobs, in press). The receptor array is composed of $\sim 2000$ mechanoreceptors, all of which are directionally selective and each of which is innervated by a single sensory receptor. Afferents are very broadly tuned to air currents, and their tuning curves overlap extensively. The afferents from these receptors projcct into the terminal abdominal ganglion to form an ordered repre-

\footnotetext{
Received April 28, 1995; revised Sept. 19, 1995; accepted Oct. 19, 1995.

This work was supported by National Institutes of Health Grant R29-NS29847 to G.A.J. We thank J. Miller, C. Boettiger, H. Clague, C. Doan, B. Girish, M. Landolfa, J. Levin, L. Osborne, C. Roddey, and T. Trover for many important and insightful discussions of this work. We express special thanks to John Miller for critically reading this manuscript.

Correspondence should be addressed to Dr. Gwen Jacobs, LSA, Box 6, Department of Molecular and Cell Biology, University of California, Berkeley, CA 94720.

Dr. Theunissen's present address: Department of Physiology, University of California, P.O. Box 0444, San Francisco, CA 94143-0444.

Copyright (C) 1996 Society for Neuroscience $\quad 0270-6474 / 96 / 160769-16 \$ 05.00 / 0$
}

sentation of air current direction (Bacon and Murphey, 1984; Walthall and Murphey, 1986). Previous studies have shown that (1) the distribution of directional selectivities among the receptor population is highly nonuniform, and (2) air current direction is not represented in a continuous topographic manner on the receptor epithelium. Thus, a continuous and uniform internal representation of air current direction could not be achieved via a direct topographic mapping of the receptor afferents into the CNS.

We addressed several specific questions regarding the functional organization of the primary afferent projection to the CNS. (1) How is a one-dimensional functional parameter (direction) mapped by an ensemble of primary afferents in three dimensions in the terminal ganglion? Is direction mapped continuously? (2) Is the degree of nonuniformity in the representation of stimulus direction seen in the receptor distribution maintained or modified within the functional map? (3) What are the functional consequences of any observed anatomical nonuniformity on the functional representation of direction? (4) What spatial patterns of activity would be predicted to emerge from the structural organization of the map?

To address these questions, two characteristics of a representative sample of identified afferents were measured: (1) the optimal stimulus direction for each afferent, and (2) the spatial distribution of each afferent's synaptic sites within the CNS. This information was incorporated into a database that preserves accurate information about the anatomical and functional relationships between the neurons within the ensemble. Algorithms were applied to map a physiological function onto the continuous distribution of synaptic terminals in the map. Quantitative and testable predictions then were made regarding ensemble response patterns within the map. 


\section{MATERIALS AND METHODS}

Development of a database of identified neurons. All of the analyses presented here were based on the development of a large database of anatomical reconstructions of sensory afferent terminal arborizations (Troyer et al., 1994). The database consisted of the anatomical reconstructions of 5 examples each of 12 different identified sensory afferterts. The 12 sensory afferents were chosen to span the entire range of directional tuning seen in this system. All 12 afferents also were associated with the longest mechanoreceptors and, therefore, had similar intensity and dynamical sensitivities to air current stimuli. For clarity, the methods associated with building this functional map have been divided into three parts: (1) the histological methods used to stain the sensory afferents; (2) the physiological methods used to determine the optimal stimulus direction of each afferent type; and (3) the analytical methods with which we quantified the organization of the map.

Histology and computer reconstruction techniques. For these studies, adult female crickets were used within $24 \mathrm{hr}$ of their imaginal molt (Bassett's Cricket Ranch, Visalia, CA). Individual sensory afferents were stained with cobalt and were silver-intensified according to the method described by Johnson and Murphey (1985) and as modified by Jacobs and Nevin (1991).

The three-dimensional reconstruction techniques used to digitize individual stained afferents were developed in our laboratory and have been described in detail elsewhere (Jacobs and Nevin, 1991). The five samples of each identified afferent, each taken from a different animal, were scaled to a common standard to obtain a valid statistical sample. To perform this task, a set of reproducible fiducial points were used that included the outline of the ganglion as well as internal markers. Details of this procedure were presented in a previous study (Jacobs and Nevin, 1991).

Measurement of mechanoreceptor functional parameters. Individual afferents have been shown to be uniquely identifiable from animal to animal because of their relatively invariant functional and anatomical characteristics. The optimal stimulus direction of each mechanoreceptor was obtained from the location and movement axis of the filiform hair on the cercus and from the orientation of the cerci with respect to the animal's body. The lengths, locations, and movement axis angles of the 12 identified filiform hairs were measured by direct observation through a dissecting microscope. Each hair's length was measured with a calibrated ocular micrometer. Its location was determined by measuring the circumferential angle of the hair on the cercus relative to the topmost (dorsal) point. The angle of each hair's movement axis with respect to the cercal axis was measured through the microscope with an ocular protractor. This angle was measured easily by blowing gently on the cercus while observing the deflection of the hair through the dissecting microscope. To determine the experimental measurement error associated with use of the ocular protractor, 12 direction measurements were made of the same identified hair on each of 6 different animals. The distribution of repeated measurements had an SD of $<2.5^{\circ}$. The angle with respect to the cercal axis then was translated into the directional angle with respect to the cricket's longitudinal axis by taking into account the angle between the cerci and the body of the cricket as described in detail previously (Landolfa and Jacobs, 1995).

Each receptor neuron showed a preferred direction or "polarity" in its responsiveness, i.e., deflection of the hair in one direction (within its movement axis) increased the spike rate, and deflection in the opposite direction decreased the spike rate. Although the movement plane of each hair was directly observable through the dissecting microscope, there were no external features that could be used to determine reliably the receptor cell's polarity within the movement axis. Therefore, an extracellular recording technique described by Bacon and Murphey (1984) was used to identify each hair's preferred direction.

Quantitative analysis techniques. We developed a general methodology to quantify the spatial distribution of the surface area of terminal varicosities of the sensory afferents. The measures are based on the expected distribution of membrane surface area of the varicosities within the three-dimensional volume of the terminal ganglion. The first task was to estimate a "surface-density" function for each of the 12 identified afferents. These three-dimensional space-filling density functions will be referred to subsequently as density clouds. A set of measures can be calculated from a density function to describe the location and spatial extent of the arborization of a single afferent, and to quantify the spatial segregation or overlap beiween the arborizations from diflerent afferents. The functional properties associated with any defined volume of the map then can be calculated from the overlapping density functions of the afferents within that volume, as determined by the colocalization of their varicosities. All calculations were done numerically. For that purpose, the three-dimensional space was discretized into voxels. The size of the voxels was determined by the accuracy required for the density estimation as explained below. The voxels were indexed with $v$ for a total of $N_{v}$ voxels. The volume of a single voxel was $l^{3}$, where $l$ was the size of one the sides of the voxel cube. The density measures in discrete form will be written as $\delta_{v}$ and will be taken as the integrated density function over the volume of the voxel.

Notation. The following notation and definitions will be used:

$X=(x, y, z)$ location of a point in the three-dimensional space of the neuropil of the terminal ganglion;

$\left\{X_{i j}\right\}$ set of locations of the varicosities for a given afferent type. For each type there are $N_{J}=5$ samples and $N_{i} \sim 500$ varicosities per sample;

$d\left(X_{i j}\right)$ the measured density amplitude of the surface area for the varicusity at $X_{i j}$. The amplitude of the surface area is taken as the square of the diameter of the varicosity;

$\delta(X)$ the true density function for a single afferent type;

$\tilde{\delta}(X)$ the estimated density function for a single afferent type; $s$ the "spread" or SD of the Gaussian kernel used in the density estimation technique.

The following notation applies to the discrete measures:

$N_{v}$ the number of voxels;

$l$ the size of one side of a voxel;

$X_{v}$ the position of the voxel;

$\tilde{\delta}_{y}$ the estimated mean density function integrated over the volume of the voxel.

To measure the variance in the overall density of output sites from five different samples taken from five different animals, we also estimated the density for each given sample, which we called $\tilde{\delta}_{j}$ and $\tilde{\delta}_{j}$.

Estimating the density function. Each sample set of five afferent reconstructions consisted of many varicosities $(-500 \times 5=2500$ per afferent type), and each varicosity had a specific diameter and a specific $x, y, z$ location in three-dimensional space. It was necessary to convert each of these distributions of discrete sample points into a continuous function representing the probabilistic density of the varicosities of a given identified afferent within the glomerulus of the cricket. To do so, the statistical techniques of density estimation were used. Density-estimation methods allow for optimal interpolation between a set of such singular sampling points to obtain a continuous representation. In our case, the transformation was achieved by deriving a function (called a kernel) which, when convolved with our discrete data set, would give the best statistical estimate of the actual continuous density function. We chose Gaussianunit kernels and used the least-square cross-validation technique to find their optimal width. This method has been shown to produce estimates that are far superior to those obtained from histograms (Silverman, 1986).

The estimated density function thus could be written as:

$$
\begin{aligned}
\tilde{\delta}(X)=\frac{1}{N_{j}} \sum_{j} \tilde{\delta}_{j}(X) \\
\quad \tilde{\delta}_{j}(X)=\sum_{i} \frac{d\left(X_{i j}\right)}{s^{3}(2 \pi)^{3 / 2}} \exp \left(-\frac{1}{2 s^{2}}\left(X-X_{i j}\right)^{T}\left(X-X_{i j}\right)\right),
\end{aligned}
$$

where $s$ is the spread or width of the three-dimensional Gaussian density function. To find the best approximation to the actual density, we found the value for $s$ that minimizes:

$E=\int(\delta(X)-\tilde{\delta}(X))^{2} d V$ or equivalently $E_{0}=\int \tilde{\delta}^{2} d V-2 \int \delta \bar{\delta} d V$

The actual distribution $\delta(X)$ was unknown, but it has been shown recently (Silverman, 1986) that the second term can be approximated by:

$$
\int \delta \tilde{\delta} d V \approx \frac{1}{N_{j}} \sum_{i j} d\left(X_{i j}\right) \cdot \tilde{\delta}_{-j}
$$




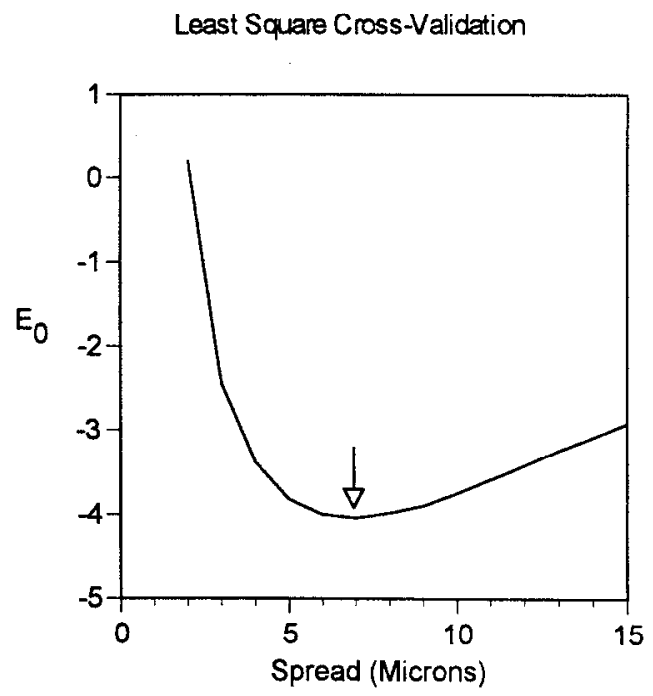

Figure 1. Determination of the optimal spread value for kernels used in quantifying the distribution of synaptic varicosities in the ganglion. Mean crror as a function of width of the Gaussian kernels was used to estimate the density function. Gaussian-shaped kernels and the least-square crossvalidation technique were used to find the optimal width of the kernels. The spread value corresponds to the width of the Gaussian function that was used. We calculated $E_{0}$ for each of the 12 afferent types for spread values ranging from 2 to $15 \mu \mathrm{m}$. The plot shows the mean error as a function of spread. On average, the minimum error was found for a spread value of $7 \mu \mathrm{m}$.

where $\tilde{\delta}_{-j}$ is the estimated density taking all of the points in the sampling set $\left\{X_{i j}\right\}$ except for all of the points belonging to the particular sample $j$.

$E_{0}$ was calculated by discretizing the volume with a set of voxels. The size of the voxel, $l$, had to be small enough so that the estimation of the integrals was accurate. However, a larger voxel would save computational time. We found that taking $l=3 / 5 s$ gave the desired accuracy, in the sense that the results did not change significantly for smaller values of $l$. The discretized form of the error $E_{0}$ is:

$$
E_{0}=\sum_{v}\left(\frac{\tilde{\delta}_{v}}{l^{3}}\right)^{2} \cdot l^{3}-2 \frac{1}{N_{j}} \sum_{j v} \frac{\left(\tilde{\delta}_{v}-\tilde{\delta}_{v j}\right)}{l^{3}} \cdot d\left(X_{v}\right),
$$

where $d\left(X_{\mathrm{v}}\right)$ is zero unless one of the $X_{i j}$ happened to fall in voxel $v$, in which case $d\left(X_{v}\right)=d\left(X_{i j}\right)$.

$E_{0}$ was calculated for each of the 12 identified afferents for spread values ranging from ? to $15 \mu \mathrm{m}$. The average mean error as a function of spread is shown in Figure 1. On average, the minimum error was found for a spread value of $7 \mu \mathrm{m}$; this value was used in all subsequent calculations.

Calculation of the center of mass of the density distribution. A first-order measure of the global location of a density cloud can be obtained by calculating its center of mass. The center of mass $X_{c m}=\left(x_{c m}, y_{c m}, z_{c m}\right)$ is found for a given density function by:

$$
x_{c m}=\frac{\sum_{v} x_{v} \cdot \tilde{\delta}_{v}}{\sum_{v} \tilde{\delta}_{v}}, \quad y_{c m}=\frac{\sum_{v} y_{v} \cdot \tilde{\delta}_{v}}{\sum_{\nu} \tilde{\delta}_{v}}, \quad z_{c m}=\frac{\sum_{v} z_{v} \cdot \tilde{\delta}_{v}}{\sum_{v} \tilde{\delta}_{v}} .
$$

Measures to compare the output density functions of two identified afferents. Two different metrics were used to characterize the segregation between density clouds from different identified afferents: the distance between their centers of mass, and their anatomical overlap. The distance between the centers of mass is an approximate measure of the segregation of the two density clouds, because it measures the separation of two complex distributions, the spatial attributes of which have been collapsed to a single point. The distance between centers of mass is simply the Euclidean distance between the two points.

The measure of overlap, however, makes a point-wise comparison of two clouds and takes into account all moments of the density distributions. The overlap between two afferent arborizations can be thought of as their intersection, and this was determined by comparing the values of

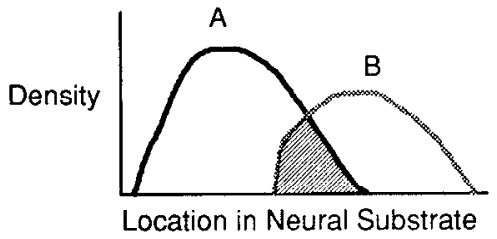

Figure 2. Schematic diagram showing the density and the anatomical overlap of two afferents in one-dimensional anatomical space. The area of overlap is shown as the hashed region. The combined area is the area under both curves, with the overlap area counted only once.

their associated density functions at each point in space. Defining $\tilde{\delta}_{A}(X)$ as the density of output sites for afferent type $A$ and $\tilde{\delta}_{B}(X)$ as the density of output sites for afferent type $B$, the overlap amplitude and the combined amplitude at point $X$, defined by $O_{A B}(X)$ and $C_{A B}(X)$, where

$$
O_{A B}(X)=\tilde{\delta}_{A}(X) \text { and } C_{A B}(X)=\tilde{\delta}_{B}(X), \quad \text { if } \quad \tilde{\delta}_{A}(X)<\tilde{\delta}_{B}(X)
$$

and

$$
O_{A B}(X)=\tilde{\delta}_{B}(X) \quad \text { and } \quad C_{A B}(X)=\tilde{\delta}_{A}(X), \quad \text { if } \quad \tilde{\delta}_{A}(X)>\tilde{\delta}_{B}(X) .
$$

The combined amplitude can be thought of as the union of the two clouds. The total overlap amplitude and the combined amplitude between type $A$ and type $B$ is given by:

$$
\bar{O}_{A B}=\int O_{A B}(X) d V \text { and } \bar{C}_{A B}=\int C_{A B}(X) d V
$$

We then define the pereent overlap (which we will refer to subsequently as the overlap) between identified neurons $\mathrm{A}$ and $\mathrm{B}$ as:

$$
\operatorname{Pov}_{A B}=100 \times \frac{\bar{O}_{A B}}{\bar{C}_{A B}} .
$$

When the density distributions are normalized, they can be thought of as being probability density functions. Therefore, this measure of percent overlap is equivalent to the mean probability that a volume of space was occupied by both type A and type B afferents if it contained either type A or type B afferents. Figure 2 illustrates these measures schematically for two different one-dimensional density functions.

Measures of significance for estimates of overlaps and distances between centers of mass. As described above, five samples of each type of afferent were reconstructed for these studies. To estimate the statistical significance for our calculations of the above measures when applied to individual samples of the same identified afferent, the variance and SE were estimated the usual way.

For the calculations involving comparisons between two different identified afferents (with different directional tuning), the objects being compared were actually the two different mean density clouds from all five samples for each of the two identified afferents. To estimate the sampling error for such comparative measures, we used the "jack-knife" resampling technique (Efron, 1982). A measure of the variance in the measurement can be estimated using Tukey's formula:

$$
\operatorname{Var}=\frac{n-1}{n} \sum_{j}(\Theta(j)-\Theta(\cdot))^{2},
$$

where $n$ is the number of samples, $j$ indexes over all samples, $\Theta$ represents the value of the relevant measure (i.e., either the percent overlap or the distance between centers of mass), $\Theta(j)$ is the value when the $j$ th sample is deleted, and $\Theta(\cdot)$ is the average of all $\Theta(j)$. The square root of this variance is an estimate of the SE of the measure $\Theta$.

"Self-overlap" between afferents of the same type. To estimate the similarity between the five different samples of a given afferent type, a measure of self-overlap was also defined based on the same jack-knife resampling technique. The calculation presented above was repeated for two identical density clouds from the same afferent type. The mean overlap obtained by averaging all of the overlaps with one sample deleted (i.e., $\Theta(\cdot)$, where $\Theta$ is the overlap measure) was taken as our measure of self overlap. The variance obtained by Tukey's formula also was calcu- 
lated and taken as an additional measure of the similarity among samples of a given afferent type.

Direction clouds. An essential goal of this work was to describe the functional organization of this ensemble of neurons as a single global entity: the neural map. To do this, it was necessary to derive a method for projecting a functional property (in this case, direction selectivity) onto the structural database of afferent neurons. The structural data consisted of the terminal arborizations of the afferent neurons, and the functional data consisted of the directional tuning curves of those same neurons. The directional tuning curves of all of the afferents in our database were identical in shape and could be characterized fully by a two-dimensional vector corresponding to the horizontal wind direction that elicited the maximum response of the neuron (i.e., the direction corresponding to the peak of the tuning curve). The functional characteristic of any region of the neural substrate then could be defined by the tuning curve that would be obtained by a weighted sum of all tuning curves from the afferents having output sites within that particular region of neuropil. The weights in that calculation were taken as the expected density of output sites for each afferent type. In the case of identical cosine-shaped afferent tuning curves, the resulting net tuning curve within any region also had this same cosine shape and, likewise, could be characterized fully by a twodimensional vector. This vector corresponded to the direction of horizontal air displacement that would elicit the maximum summed activity from ail of the output sites at that location in the neural substrate. In this case, the calculation of the functional property of a region in the map could be reduced to a simple weighted vector sum. The mathematical steps for this calculation are described below.

Each afferent type contributed a density of output sites at point $X$, which we have defined as $\delta(X)$. Each afferent type was maximally sensitive to a particular direction in the horizontal plane, which could be described by a two-dimensional unit vector with a direction $\theta$. The functional contribution of that given afferent to the point $X$, therefore, could be described by a vector of magnitude $\tilde{\delta}(X)$ and direction $\theta$. The overall functional property of point $X$ then could be represented by the vector sum of all contributions from each afferent type. The overall functional property characterizing the map at point $X$ also could be represented by a two-dimensional vector of direction $\theta(X)$ and magnitude $\Delta_{\text {all }}(X)$. We can express this relationship mathematically as:

$$
\left\{\begin{array}{l}
\Delta_{\mathrm{All}} \sin (\theta(X))=\sum_{k} \tilde{\delta}_{k} \sin \left(\theta_{k}\right) \\
\Delta_{\mathrm{All}} \cos (\theta(X))=\sum_{k} \vec{\delta}_{k} \cos \left(0_{k}\right),
\end{array}\right.
$$

where $k$ is the index over our sample of identified afferents.

To visualize the functional property of each location of the map space, we discretized the horizontal directional sensitivity into 16 bins of $22^{\circ}$ each. Each point $X$ within the glomerulus belonged to 1 of the 16 directional bins, as dictated by its directional sensitivity $\theta(X)$. Each point $X$ also had a given density $\Delta_{\text {all }}(X)$. The resulting image is of 16 nonoverlapping directional clouds, color-coded according to direction and having a density given by $\Delta_{\text {illl }}$ (see Fig. 10C).

\section{RESULTS}

The primary afferent input to the cercal sensory system comes from an ensemble of mechanosensory afferents that innervate filiform hairs on two sensory appendages, called cerci, located at the rear of the animal's abdomen (Fig. $3 A$ ). Filiform hairs are constrained by a cuticular socket to move in a single plane with respect to the cercal surface (Fig. $3 B$ ). The receptors are grouped on the cerci in two large groups: hairs that move transversely with respect to the cercus are located on the dorsal and ventral surfaces, and hairs that move longitudinally are located on the lateral and medial aspects of the cercus (Palka et al., 1977; Bacon and Murphey, 1984; Walthall and Murphey, 1986). Afferents are broadly tuned to air currents directed at the animal's body, and their tuning curves can be approximated with a cosine function (Landolfa and Miller, 1995) (Fig. 3D). Afferents are active under ambient air conditions, and their firing rate is modulated up or down as a function of air current (stimulus) direction. Each mechanoreceptor is innervated by a single afferent that projects its axon to the terminal abdominal ganglion through the cercal nerve (Fig. 3C). Primary afferents provide direct excitatory input to an ensemble of sensory interneurons that project their axons out of the terminal ganglion to higher centers of the nervous system.

\section{Selection of the representative sample of filiform hairs}

The goal of this study was to understand, at a quantitative level, the anatomical constraints on the functional organization of the map of stimulus direction in the cricket cercal sensory system. Our approach was to reconstruct a probabilistic atlas of the entire map from individual identified afferents stained in many different animals. Because afferents in the cercal system are uniquely identifiable (Palka et al., 1977; Walthall and Murphey, 1986; Landolfa and Jacobs, 1995), the problem of ensuring a representative sample of receptors for synthesis of a global map was reduced to the selection of an appropriate set of identified filiform hairs.

A sample of identified afferents was chosen to build the atlas using the following three criteria. (1) All afferents had the same dynamic sensitivity characteristics. Dynamic sensitivity is highly correlated with receptor hair length, so the sample was limited to afferents innervating the longest mechanoreceptors on the cercus (1500-1900 $\mu \mathrm{m})$. Previous studies have shown that afferents associated with hairs of different lengths and ages have different functional properties and are sensitive to different intensity and frequency ranges (Shimozawa and Kanou, 1984b; Kamper and Kleindienst, 1990; Roddey and Jacobs, in press). Hair length also is correlated with age of the sensory neuron, so limiting the sample in this way ensured that all cells were the same developmental age (Murphey et al., 1980).

(2) The sample of afferents represented the intrinsic distribution of receptors across all stimulus directions. In a previous study (Landolfa and Jacobs, 1995), the distribution of receptors with respect to directional sensitivity was shown to be continuous but nonuniform. Our sample was chosen to reflect that distribution. Figure 4 shows the sample of cells used in this paper with respect to the overall distribution of receptor directional sensitivities.

(3) The sample included cells at many cercal locations to ensure that all circumferential locations on the cercus were represented. The distribution of the sample with respect to cercal location is shown in Figure $5 A$.

Figure 5, $A$ and $B$, also shows the change in directional tuning as a function of the circumferential location of the receptor on the cercus. Previous work (Walthall and Murphey, 1986; Landolfa and Jacobs, 1995) showed that directional tuning changed smoothly with cercal position around the ventral surface of the cercus. However, at all other circumferential locations, tuning changes abruptly with circumferential angle.

Our sample consisted of a total of 60 identified sensory cells ( $n$ $=5$ for each of 12 different identified neurons). The terminal arborizations of the identified neurons are shown in Figure 6. Each image is a computer reconstruction of onc example of that identified neuron, shown in dorsal view. Each reconstruction is shown inside a box that indicates the spatial extent of the left cercal glomerulus in dorsal view (Fig. 6A). The right border of the box indicates the midline of the ganglion. An arrow showing the optimal wind stimulus direction of each cell in body coordinates is shown next to the reconstructions. Each afferent was given a number corresponding to the cercal locations described in Walthall and Murphey (1986). All identified afferents, except \#12, arborized ipsilaterally in the ganglion. Neuron \#12 had a bilateral arborization in the ganglion (Murphey and Lemere, 1984). 

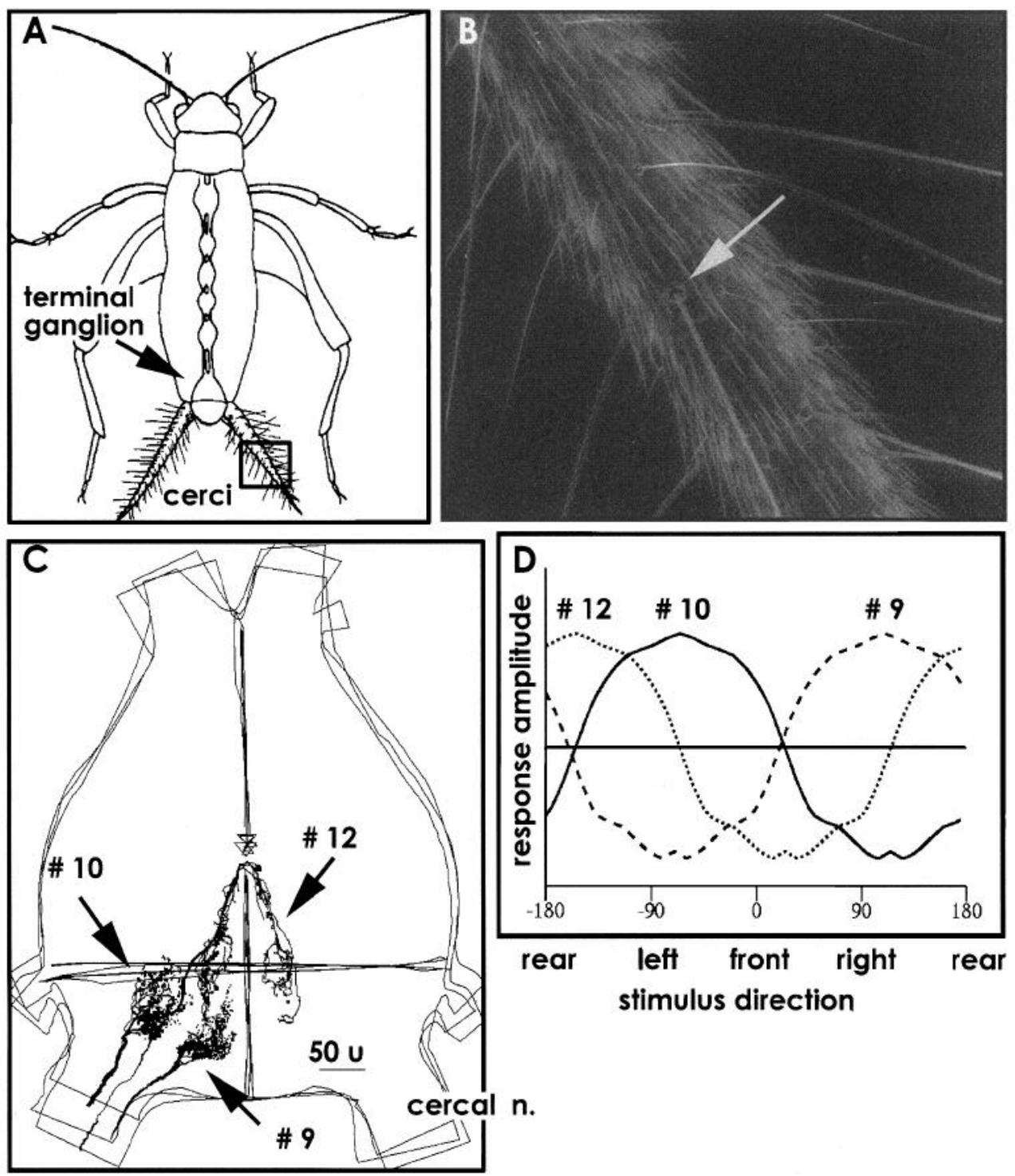

\section{Determination of the inter-animal variability in the structure of identified afferent terminal arborizations}

How similar are the terminal arborizations of identified sensory afferents between animals? To address this question, we examined the anatomical differences between five individual examples of each identified afferent. Visual inspection suggested that there was significant variability in axon trajectory and in the number of primary and secondary branches within different examples of the same identified neuron. However, it appeared that the terminal varicosities of different examples of the same identified sensory cells occupied a similar target area in the terminal ganglion in different animals. To quantify these observations, the ensemble of output sites from all five samples of each identified afferent was used to estimate the probability density function (density cloud) for varicosities within the glomerulus (see Materials and Methods). Density clouds for each identified afferent were obtained by calculating the fraction of the overall density cloud that belonged to each sample neuron (i.e., the sample density cloud, see Materials and Methods).

We examined the global location of each afferent and its density distribution in the neuropil by calculating two independent mea-
Figure 3. Schematic diagram of the cricket cercal sensory system. $A$, Diagram of a cricket showing the location of the cerci, the abdominal nervous system, and the terminal abdominal ganglion (arrow). The inset box on the right cercus indicates the area of the cercus shown in $B$. $B$, Environmental scanning electron micrograph of the distal part of a cercus from an adult cricket. Filiform hair mechanoreceptors can be seen as long, hair-like structures lodged in cuticular sockets on the cercus. The arrow indicates a socket. Note that the hairs are distributed uniformly over the cercal epithelium and project from the cercus from many different angles. Magnification, $130 \times . C$, Computer reconstruction of the terminal arborizations of three identified sensory afferents shown within the outline of terminal abdominal ganglion. Each terminal arbor occupies a specific location within the terminal ganglion. The numbers denote a specific identified afferent, as shown in Figure 6. Scale bar, $50 \mu \mathrm{m}$. $D$, Directional tuning curves of the identified afferents shown in $C$. The tuning curve of mechanosensory afferents can be approximated with a cosine function. The peak of the curve indicates the preferred stimulus direction for each afferent. The horizontal line indicates the spontaneous firing level of the afferent, which is modulated up or down as a function of stimulus direction. Note that identified afferents \#9 and $\# 10$ are tuned to stimulus directions $180^{\circ}$ apart. They occupy spatially segregated positions in the ganglion, as shown in $C$.

sures from the sample density clouds: the amount of anatomical overlap of the different individuals within the sample set, and the distance between their centers of mass. The center of mass of the density cloud is a measure of the global location of that cloud (indicated in Fig. 7 by a cross near the center of the density cloud). The amount of anatomical overlap between samples is a measure of the similarity in shape and density of the terminal arbor across animals. These data are shown for one identified afferent in Figure 7. Note that in the three samples shown, the clouds have similar shapes and locations. Their centers of mass are positioned to within $\sim 13 \mu \mathrm{m}$ of one another.

Table 1 shows the mean distance between centers of mass and variance between the samples of density clouds of each identified afferent. The mean distance between centers of mass across all receptors was $17 \mu \mathrm{m}$. This is a small distance compared with the overall size of the glomerulus $\left(120 \times 160 \times 280 \mu^{3}\right)$ and with the overall extent of typical sample density clouds. The spatial extent of each density cloud was large with respect to the entire volume of the map. On average, a density cloud occupied $32 \%(\mathrm{SD}=5 \%)$ of the entire volume of the map defined by the ensemble of identified afferents. 
A

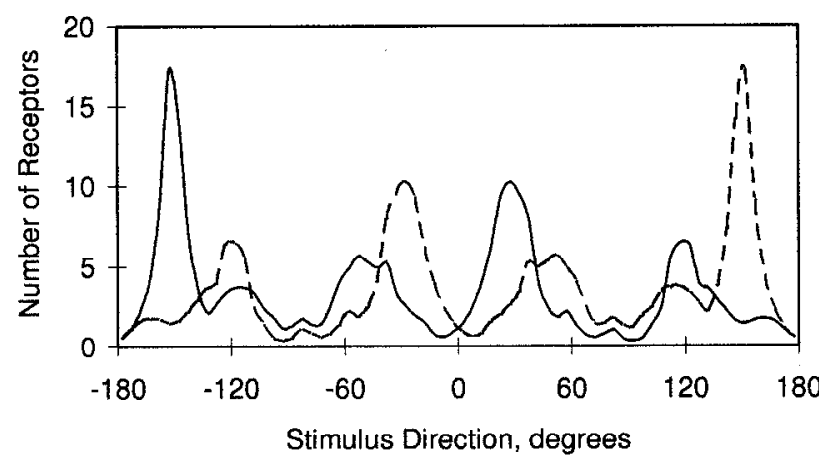

rear left front right rear

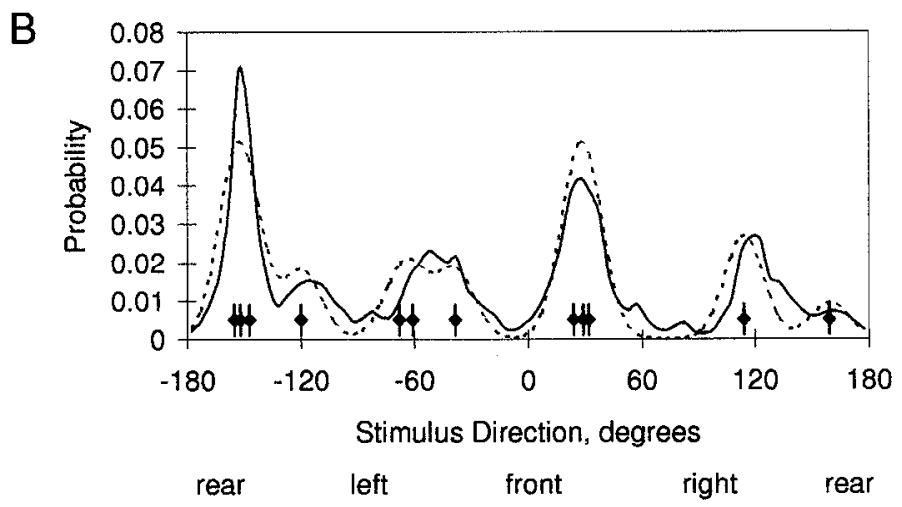

Figure 4. Distribution of receptor types according to directional sensitivity. $A$, The distribution of filiform hair directions with respect to the crickel's body axis. The number of filiform receptors with a particular preferred direction was plotted against the stimulus direction. The distribution is shown for the left cercus as a solid line and for the right cercus as a dotted line. Data replotted from Landolfa and Jacobs (1995). B, The distribution of receptors on the left cercus compared with an estimate of the distribution that was obtained from the sample of 12 afferents used in this study. The solid line indicates the distribution of afferents on the left cercus as shown in $A$. The dotted line indicates the estimated distribution generated by convolving the peak directions of the 12 identified afferents with a Gaussian kernel. The width of the kernel was chosen to obtain the best fit with the actual distribution of afferents on the cercus. The peak directional tuning of each identified afferent is shown as a small diamond. The close match indicated that our sample is a good representation of the overall distribution of directional sensitivities in the receptor array.

The mean total surface area of the varicosities and the variance across samples also are shown in Table 1. Both measures of variability are small, suggesting that the gross location and number of output sites for a given identified afferent were preserved from animal to animal. Note that the total surface area of the arborizations of the different afferents were all approximatcly equal $\left(1188 \pm 237 \mu \mathrm{m}^{2}\right)$. This suggests that directional tuning associated with each afferent type had a very similar representation within the neural map.

The anatomical overlap between different examples of the same identified afferent across animals was measured by calculating the anatomical overlap between different sample density clouds derived from subsets of the samples of the same identified afferent. This gave a measure of the self-overlap. This measure of anatomical overlap was sensitive to local differences in the density distributions that were not necessarily reflected in the center of mass measure. The mean self-overlap for different examples of identi-
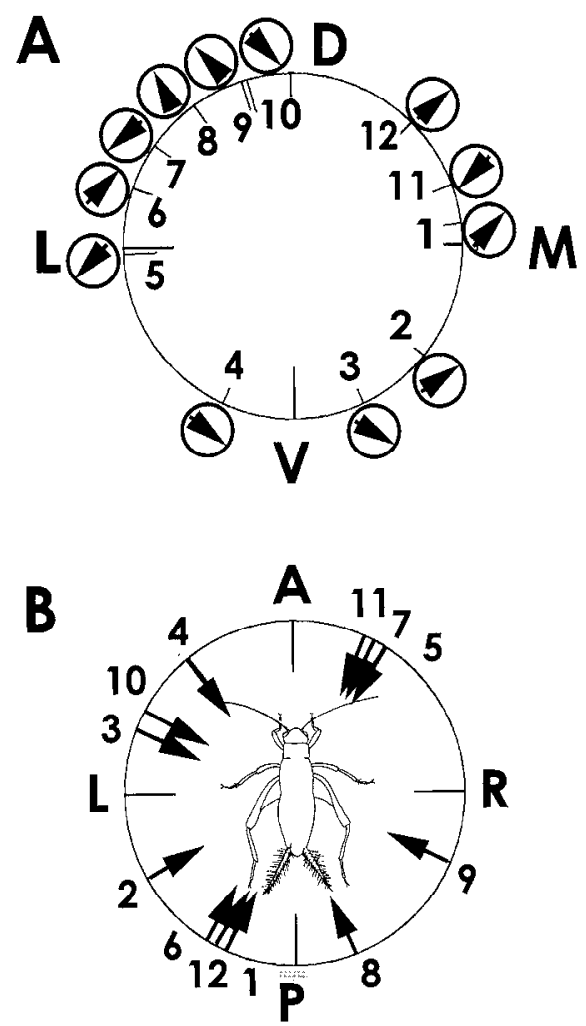

Figure 5. Circumferential location and directional tuning of identified mechanoreceptors on the left cercus. $A$, Polar plot of the circumferential location of the hairs in our sample, referenced to a cross-section through the left cercus. The circumferential location of each identified mechanoreceptor (indicated with a number) is shown along with an arrow that indicates its peak directional tuning in body coordinates. Directional tuning changes smoothly only along the ventral surface of the cercus. At all other regions, directional tuning between adjacent receptors changes abruptly wilh circunferential position: $D$, dorsal; $V$, ventral; $L$, lateral; $M$, medial. Scale is in degrees: 0 at dorsal to 180 at ventral. $B$, Preferred stimulus directions for each identified afferent with respect to the animal's body. Arrows indicate the preferred stimulus direction for each identified afferent. Note that cells with very similar directional tuning $(5,7$, and 11 or 1,6 , and 12 ) are located at many different locations on the cercus: $A$, anterior; $R$, right; $P$, posterior; $L$, left. Scale: degrees in the horizontal plane -0 toward the animal's head.

fied afferents was $96.32 \pm 9.67 \%$. The variability was small in all cases and very uniform across all types, suggesting that the target area was very well defined for each identified afferent.

These results indicate that each identified receptor type had a characteristic structure and filled a specific volume of the cercal glomerulus in all animals. No neurons were found that were out of register with the rest of the examples of the same identified neuron. Although this has never been demonstrated quantitatively before, it is in agreement with the qualitative observations of Murphey and colleagues on other identified neurons (Bacon and Murphey, 1984; Walthall and Murphey, 1986).

\section{Quantitative assessment of the relationship among directional tuning, cercal position, and degree of anatomical overlap}

In most sensory maps, primary afferents project topographically to a certain target location in the nervous system according to the position of the receptor in the epithelium (e.g., retinotopy, somatotopy, and tonotopy). Nearest-neighbor receptors in the epithelium have neighboring terminal arbors in the map. We examined 

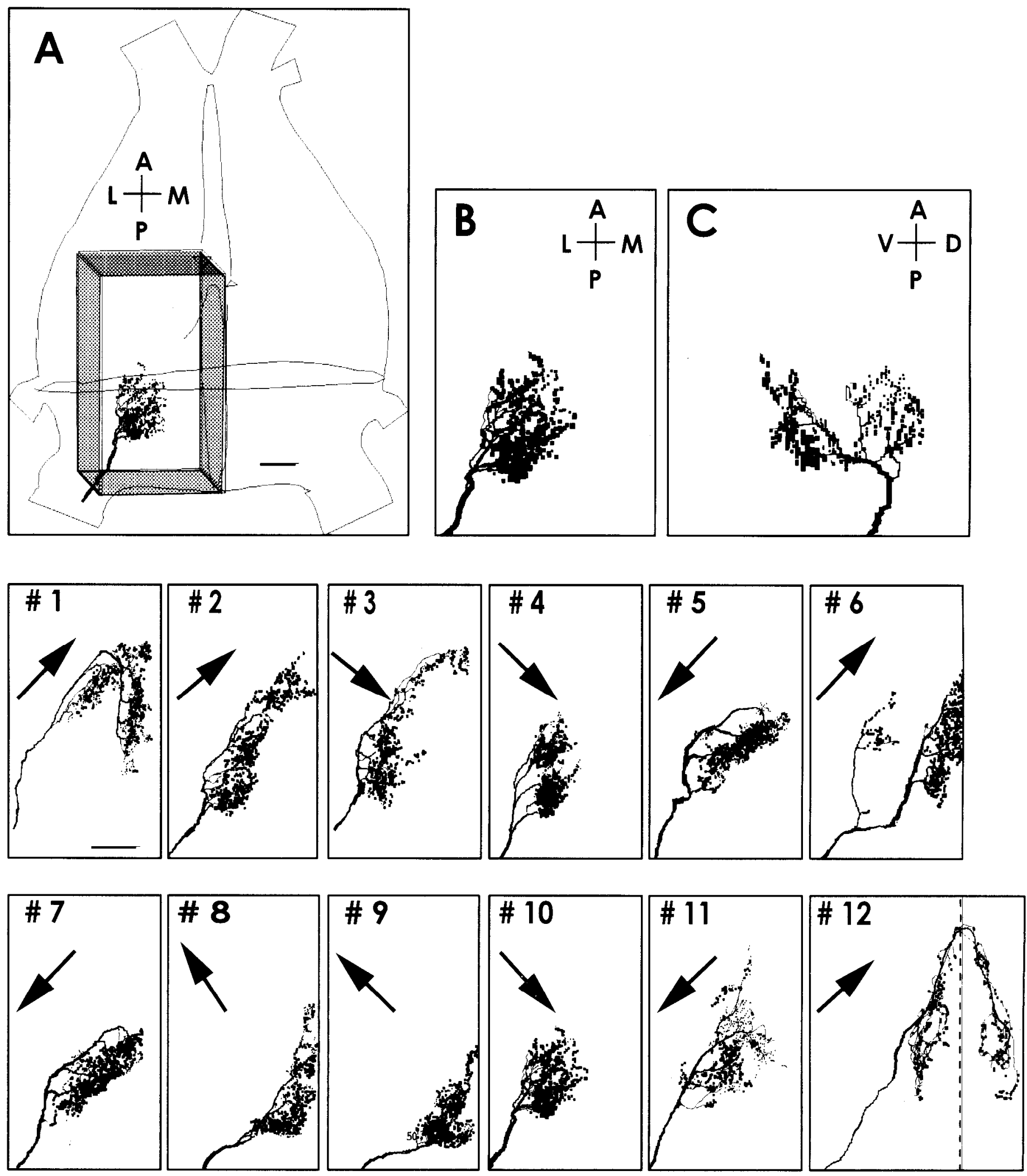

Figure 6. Terminal arborizations of 12 types of identified sensory afferents. $A$, Computer reconstruction of afferent \#10 shown with respect to the outline of the terminal ganglion. Horizontal and vertical loops around ganglion represent the ganglion outlines along those sections, which are used as fiducial features in the scaling and aligning process. The box inside the left half of the ganglion indicates the spatial extent of the cercal glomerulus. The medial and lateral faces of the box have been shaded for clarity. The image has been rotated in the sagittal plane to show the three-dimensional aspect of the box. $B$, Enlarged dorsal view of afferent \#10. The outline indicates the sides of the box as shown in $A$. $C$, Sagittal view of afferent \#10. Note that the two major branches of this cell are much more visible from this viewing angle. Bottom two panels, Computer reconstructions of all identified afferents used in this study, shown in dorsal view. The box indicates the extent of the left cercal glomerulus as in $A-C$. Arrows indicate peak sensitivity direction for each receptor. Note that cell \#12 has a branch extending across the midline of the ganglion. Scale bars, $50 \mu \mathrm{m}$. 
Figure 7. Variability in the structure of the terminal aborizations of one identified afferent across animals. In each of the top three panels, one example of identified afferent \#4 is shown in dorsal view (left) and sagittal view (middle). The probability density function of the terminal varicosity field is shown in sagittal view on the right. The cross in each image represents the center of mass of the probability distribution. Note that each probability distribution has a similar shape and position within the cercal glomerulus (indicated by the three-dimensional box). However, the axon trajectories and branching patterns are not identical across animals. Bottom panel, Stereo pair of the probability distribution shown in the right panel directly above (black dots) with the average probability distribution calculated from five examples of this cell taken from five different animals (white dots). Note that the distributions overlap to a great extent. There are some regions of only white dots, which reflects the variability between the average distribution and the individual sample. The centers of mass of the two distributions are shown with white and black crosses, respectively; the distance between them is $10 \mu \mathrm{m}$.
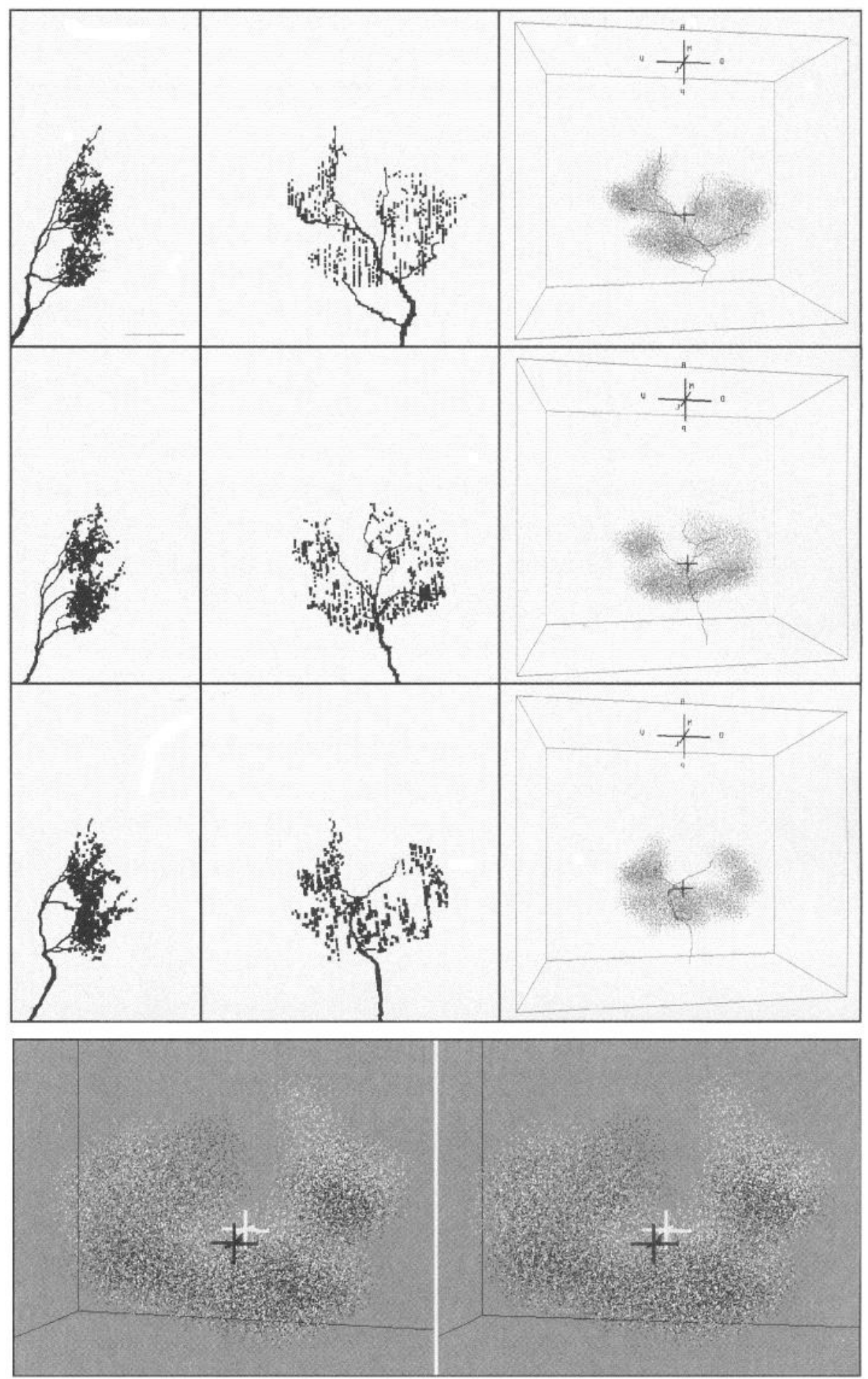

this relationship directly by calculating the amount of anatomical overlap between the terminal arbors of all pairs of afferents and plotted it as a function of the circumferential distance between the receptor pair on the cercus (Fig. $8 A$ ).

There was no significant correlation between the relative locations of two receptors on the cercus and the anatomical overlap between their terminal arbors. Thus, a continuous and uniform internal representation of air current direction could not be achieved via a direct topographic mapping of the receptor afferents into the CNS.
Alternatively, previous work has suggested that afferents were arborizing in the glomerulus according to their directional tuning characteristics (Bacon and Murphey, 1984; Walthall and Murphey, 1986). To test this idea quantitatively, anatomical overlap was plotted as a function of the difference in peak tuning between pairs of afferents (Fig. $8 B$ ). The difference in directional tuning between receptors was measured as the angular distance between the peaks of their tuning curves. The percentage of anatomical overlap decreased as the difference in directional tuning increased. The percentage overlap decreased up to separations in 
Table 1. Anatomical characteristics of identified sensory afferents

\begin{tabular}{lllll}
$\begin{array}{l}\text { Afferent } \\
\text { type }\end{array}$ & $\begin{array}{l}\text { Total area } \\
\left(\mu^{2}\right)\end{array}$ & SD area & $\begin{array}{l}\text { Distance } \\
\text { CM }(\mu \mathrm{m})\end{array}$ & SD CM \\
\hline 1 & 1029.05 & 167.42 & 15.42 & 6.34 \\
2 & 1319.75 & 266.44 & 17.51 & 5.80 \\
3 & 1113.05 & 277.81 & 18.37 & 7.70 \\
4 & 1392.86 & 147.93 & 13.22 & 4.88 \\
5 & 1052.16 & 227.04 & 15.64 & 6.75 \\
6 & 1062.83 & 273.48 & 16.61 & 7.11 \\
7 & 1314.72 & 201.66 & 12.10 & 5.85 \\
8 & 1275.67 & 340.40 & 10.58 & 2.19 \\
9 & 1254.45 & 322.84 & 20.63 & 11.77 \\
10 & 1492.97 & 265.07 & 19.02 & 5.84 \\
11 & 1072.73 & 94.93 & 11.01 & 3.80 \\
12 & 882.23 & 259.24 & 30.47 & 15.12 \\
Average & 1188.54 & 237.02 & 16.71 & 6.93
\end{tabular}

Variability in anatomical characteristics of identified afferents. For each identified type of afferent, the total area of the density cloud and the average distance between the center of mass of the density clouds for each sample were measured. See Materials and Methods for details. CM, Center of mass.

A
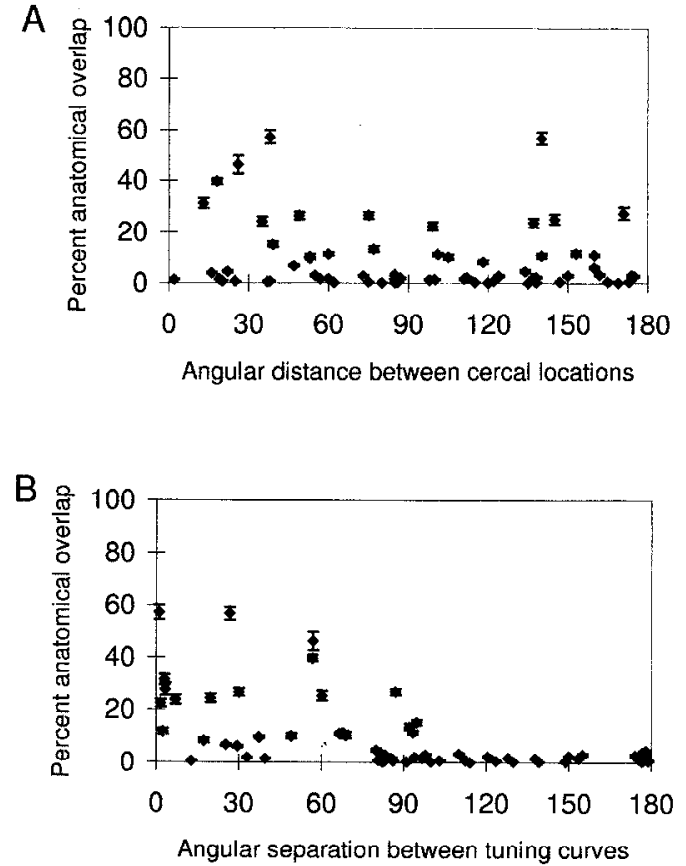

Figure 8. Relationship among directional tuning, cercal position, and anatomical overlap. $A$, The angular separation between identified receptors on the cercus was plotted as a function of the percent anatomical overlap between their terminal arborizations. Note that there is no apparent trend between the distance between two receptors on the cercus and the amount of overlap between their terminal arbors. $B$, The angular separation between the peaks of dircetional tuning curves of pairs of identified afferent types was plotted as a function of the percent anatomical overlap between average density clouds. Note that as the separation between tuning curves increases the amount of anatomical overlap decreases. There was essentially no anatomical overlap between pairs of afferents for which tuning differed by $>90^{\circ}$.

directional tuning of $\sim 90^{\circ}$. There was essentially no overlap between pairs of cells in which tuning differed by $>90^{\circ}$. It appeared that cells with similar directional tuning had overlapping terminal arborizations, and the arbors of cells tuned to different directions were spatially segregated. Directional tuning, therefore, appeared to be the primary correlate of the location of sensory neuron arbors in the ganglion. Thus, any ordered map of air current direction must be synthesized within the CNS via a substantial spatial reorganization of afferent terminals.

Figure 9 shows four examples of the correlation between directional tuning and arbor position in the ganglion. Two pairs of identified afferents had similar directional tuning ( 1 and 6,5 and 7 ), and the other two pairs were tuned to opposite stimulus directions ( 9 and 10,1 and 11 ). Afferents with opposite directional tuning were spatially segregated in the map, whereas cells with similar tuning occupied contiguous regions of space. In contrast, identified neurons that were near neighbors on the cercus (cells 9-11 and cells 5-7) did not show a consistent pattern of arborization with respect to their location in the epidermis. Cells 9-11 were all spatially segregated in the map. Cells 5 and 7 had overlapping arborizations, whereas cell 6 was segregated from both of them.

If directional tuning were the only constraint on arbor position in the CNS, then the relationship between tuning and anatomical overlap would follow a similar trend for all pairs of afferents. There are obvious exceptions to this trend. For example, cells 5 and 7 had nearly identical directional tuning and showed extensive overlap (57\%). Cells 1 and 6 also have identical tuning but showed very little overlap (5\%). However, cells 1 and 6 occupied adjoining volumes of the neural map, forming a single, extended target region. The lack of overlap in this last case would not degrade the functional representation of that stimulus direction in the glomerulus, because both cells projected to a specific functional region of the map and did not overlap with afferents tuned to other directions.

\section{Global structure of the map}

The fact that the ensemble of output sites for a given afferent type was so similar in shape and relative location from animal to animal indicated that it was possible to construct a global probabilistic atlas of the location of the output sites for the entire experimental sample.

The combined images of all reconstructed afferents in Figure $10, A$ and $B$, show how stimulus direction is mapped in the coordinate system of the ganglion. The ensemble forms a series of overlapping clouds that maintain continuity in representation of stimulus direction. In these images, the mean density cloud for each individual afferent type in the ensemble was color-coded according to its peak directional tuning angle with respect to the animal's body axis.

The entire neural map is a bilateral structure, consisting of two mirror-symmetric glomeruli, each originating primarily from the terminal arborizations of afferents innervating the ipsilateral cercus. Each glomerulus contains a "hemi-map" in which all wind directions are represented. These two hemi-maps are symmetric in shape but not identical because cells sensitive to similar directions with respect to the animal's body arborize in different relative locations on either side of the map. This functional organization is attributable to the cerci and the mechanoreceptor array being mirror images of each other. A mechanosensor on the left cercus sensitive to wind from the left has a homolog on the right cercus that is most sensitive to wind from the right. These cells arborize in mirror-image locations across the midline. Although these cells are functionally equivalent with respect to individual cerci, they are functional opposites in the animal's intrinsic coordinate system. Cells sensitive to stimulus directions along the body axis arborize in symmetrical regions of the map; 

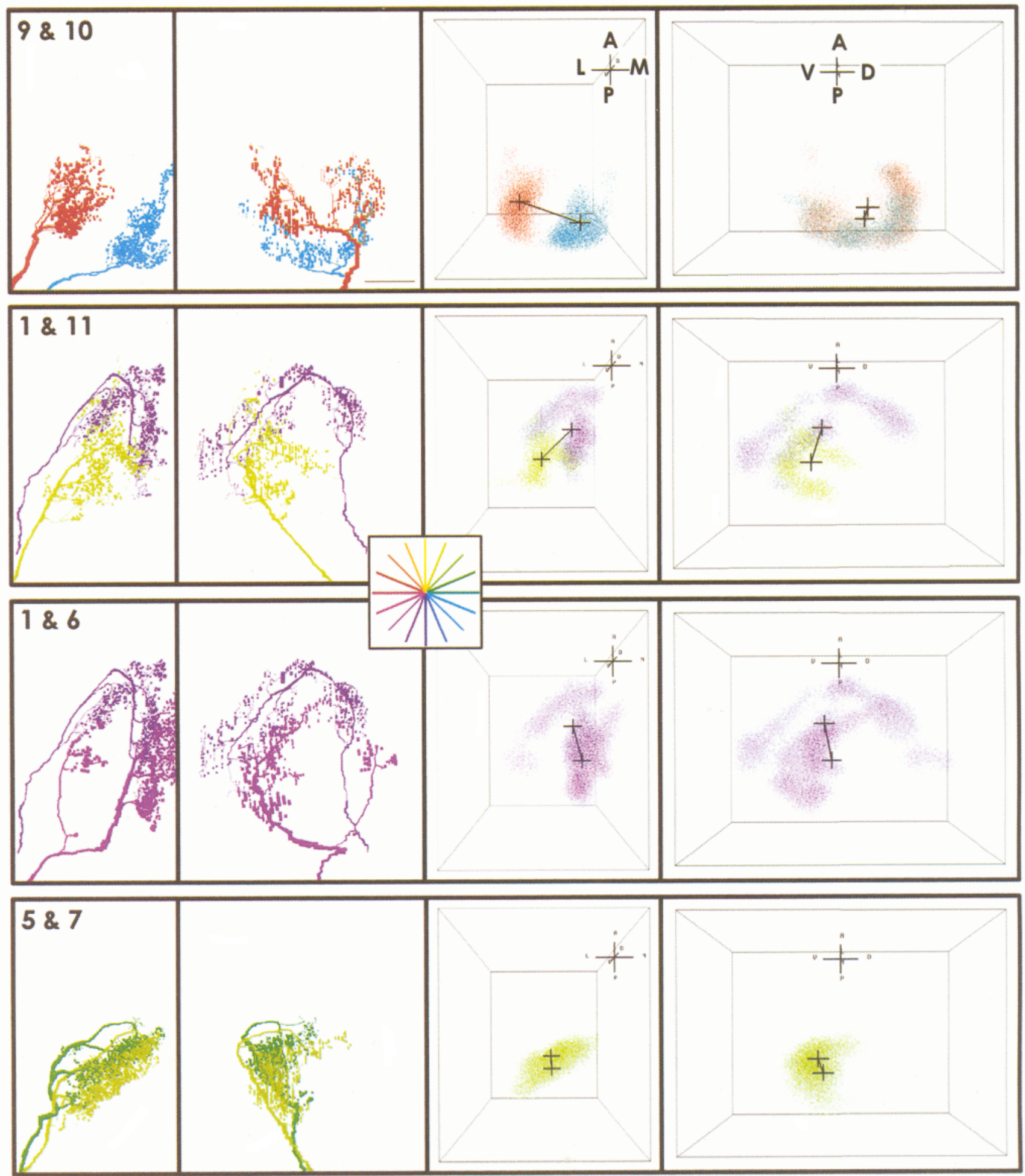

Figure 9. Anatomical relationships between identified afferents. In each panel, the two left images are dorsal and sagittal views of computer reconstructions of a pair of identified afferents. The two right images are average probability density functions of the terminal varicosities of the pair of afferents. The center of mass for each distribution is indicated with a cross. The color of each afferent and density cloud indicates its directional tuning with respect to the animal's body. The color wheel in the center of the image shows direction with respect to the animal's body in the horizontal plane. Yellow indicates a stimulus directed toward the animal's head. Cells tuned to opposite stimulus directions $(9$ and 10 or 1 and 11$)$ are spatially segregated in the glomerulus. Cells tuned to similar stimulus directions ( 1 and 6 or 5 and 7 ) occupy contiguous regions of the glomerulus. 

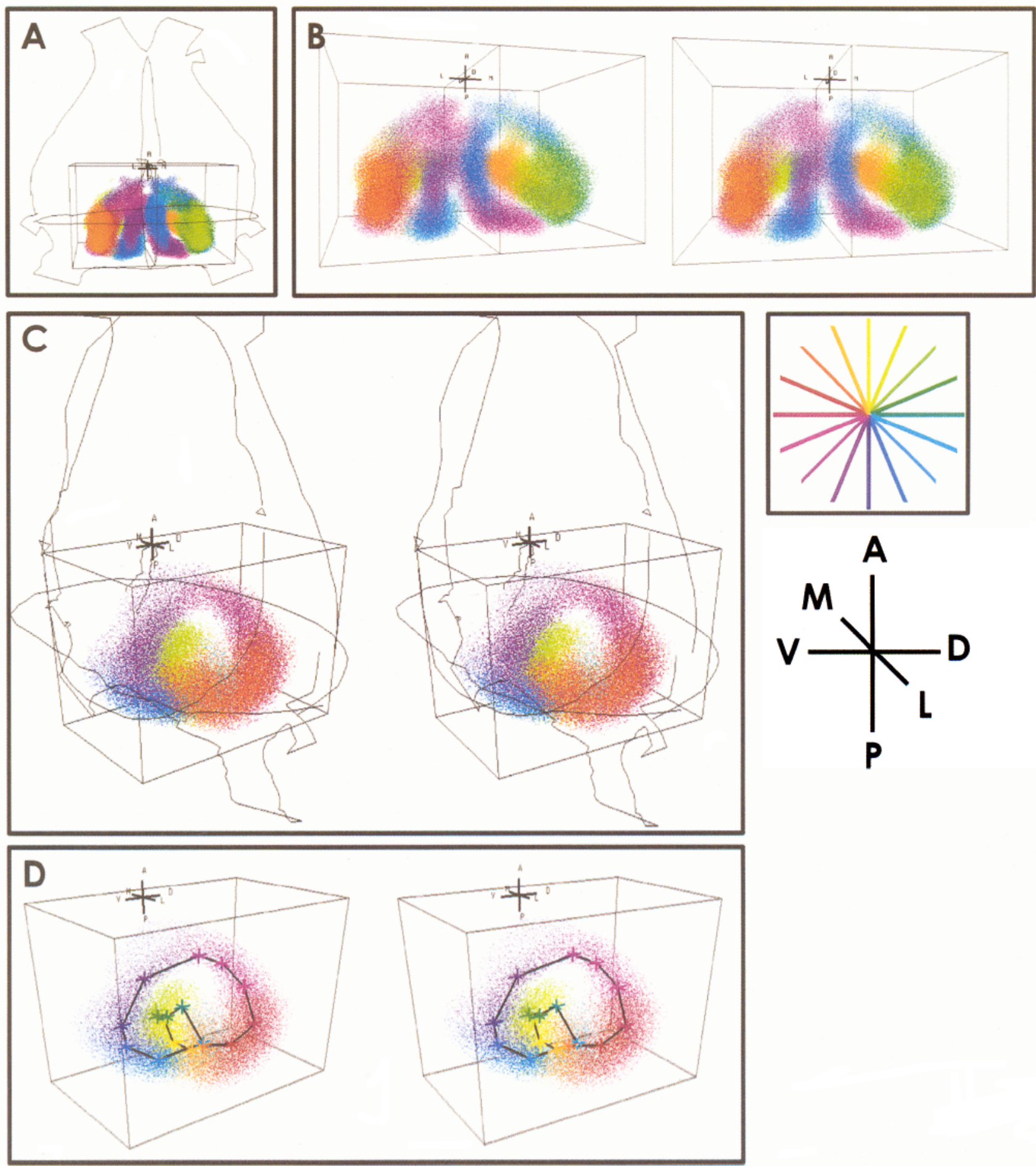

Figure 10. The functional map of stimulus direction. $A$, Image of the ensemble of sensory afferents that comprise the neural map within the terminal abdominal ganglion in dorsal view. The color of each density cloud indicates its directional tuning with respect to the animal's body. The outline indicates the limit of terminal abdominal ganglion. The color wheel at the right indicates stimulus direction with respect to the animal's body in the horizontal plane. Yellow indicates stimuli directed toward the animal's head. $B$, Stereo-pair image of the functional map shown in dorsal view, enlarged with respect to $A$. Afferents from each cercus form a hemi-map on one side of the ganglion. The two hemi-maps are mirror images of each other across the midline of the ganglion. $C$, Functional representation of stimulus direction within the neural map. Stereo-pair image of a parasagittal view of the hemi-map formed by afferents from the left cercus shown within the terminal ganglion. The viewing angle is from the ventral lateral aspect of the ganglion (as shown in the three-dimensional cross-bar in the inset). Each density cloud indicates the average probability distribution of one type of identified afferent. Color indicates directional tuning in body coordinates. The map is composed of a set of broad overlapping clouds of varicosities through which direction changes in a continuous manner. $D$, Uniform representation of stimulus direction. Stereo-pair image of the density distribution of 16 different directions formed by anatomical overlap between density clouds shown in $C$. Each region represents the vector summation of the density clouds of all afferents within that spatial region of the map. Colored crosses within each cloud indicate the center of mass of the cloud. Note that the centers of mass form a continuous contour in the three-dimensional space of the glomerulus. Viewing angle is identical to $C$. 


\begin{tabular}{|c|c|c|}
\hline $\begin{array}{l}\text { Afferent } \\
\text { type }\end{array}$ & $\begin{array}{l}\text { Self-overlap } \\
(\%)\end{array}$ & SE overlap \\
\hline 1 & 94.81 & 11.68 \\
\hline 2 & 96.42 & 9.65 \\
\hline 3 & 95.72 & 12.02 \\
\hline 4 & 97.28 & 7.04 \\
\hline 5 & 96.61 & 9.73 \\
\hline 6 & 96.60 & 9.63 \\
\hline 7 & 96.78 & 8.82 \\
\hline 8 & 95.49 & 11.11 \\
\hline 9 & 96.54 & 9.16 \\
\hline 10 & 96.48 & 9.25 \\
\hline 11 & 97.01 & 7.52 \\
\hline 12 & 96.09 & 10.47 \\
\hline Average & 96.32 & 9.67 \\
\hline
\end{tabular}

Anatomical overlap between density clouds of multiple samples of the same afferent type. Self-overlap between five samples of each identified afferent type was calculated and the mean plotted in the middle column. The third column shows the variance in self-overlap attributable to sampling errors as calculated from Tukey's formulae (see Materials and Methods).

the regions of the map representing stimulus directions from the front or rear are symmetric across the midline.

\section{Functional attributes of the map}

The ensemble of sensory afferents formed a continuous geometrical shape within the ganglion: all varicosities were clustered around a closed spiral that curved through the glomerulus, as seen in a parasagittal view of the ensemble in Figure $10 C$. The arborization of each of the 12 afferents, shown here with color coding for stimulus direction, clustered around a distinct location within the spiral. The afferents were organized according to their directional tuning, such that tuning changed continuously around the spiral.

There was a substantial degree of overlap between the density clouds of afferents with similar directional sensitivities (Figs. $8 B$, $10 C$ ). Most regions of the map contained output sites from multiple afferents with different peak directional sensitivities. Thus, the net local directional sensitivity represented at most points in the map was intermediale in value between the peak directional sensitivities of any of the afferents that projected to that region. Specifically, the net directional sensitivity represented at any location corresponded to a vector sum of the individual directional sensitivities of the cells that arborized in that region. The net directional sensitivity was represented by dividing the map into 16 regions corresponding to 16 equally spaced stimulus directions (see Materials and Methods). The resulting image is of 16 nonoverlapping "directional clouds," each color-coded according to net directional sensitivity (Fig. 10D).

This image demonstrates the relative representation of these different stimulus directions within the map. In Figure $10 D$, the black line connecting the centers of mass of each of the directional regions forms a continuous contour in the map. Thus, despite the discontinuous distribution of afferents according to their directional tuning on the cercus, the overlapping projection pattern of the output sites causes a continuous representation of stimulus direction.

\section{The spatial patterns of activity in response to specific stimuli}

It was possible to predict the steady-state spatial pattern of excitation that would be elicited within the map by air current stimuli directed at the animal from any arbitrary direction (Fig. 11). Stimuli from different directions were predicted to produce specific spatial patterns of excitatory activity within the neural map. What are the relative anatomical and functional constraints on these patterns of activity? The contribution of each afferent type to the overall pattern of activity depends on the distribution of that afferent's output sites throughout the map and the relative activation level of that afferent by the stimulus. The tuning curves for these afferents are broad and overlapping, thus any stimulus would activate a large proportion of the ensemble. The relative activation of any afferent by a stimulus from a particular direction was calculated from its directional tuning curve as the cosine of the angle between the stimulus direction and the peak direction of the associated receptor hair.

The images of specific activity patterns show the subset of the directional clouds that would be excited above their baseline levels by air currents from four orthogonal directions (Fig. 11). These images were obtained by multiplying the net peak density amplitude of a region of the map by the cosine between the peak direction of that region of the map and the direction at which the stimulus was delivered. Negative values corresponding to areas of inhibition are not shown but can be deduced from the resulting picture obtained by activating the map with stimuli from the opposite direction.

These predictions indicate that any stimulus will activate a broad region of the map by activating many afferents with overlapping arborizations. Thus, each stimulus direction is represented within the map by a pattern of activity with a unique three-dimensional shape. These activity shapes do not correspond to the anatomical structures of individual afferents, but are synthesized by the map from the combined activation of many afferents.

\section{DISCUSSION}

\section{A synthetic map formed by primary afferents}

Various schemes have been proposed for classifying sensory maps based on their structural characteristics. One scheme divides maps into two classes: projectional maps and computational (or centrally synthesized) maps (Knudsen et al., 1987; Heiligenberg, 1991; Konishi, 1991). The map of stimulus direction in the cercal system is continuous within the terminal ganglion, but definitely is not established via a point-to-point topographic mapping of the sensory epithelium onto the neural substrate. Although the directional tuning of receptors changes in a graded manner within some restricted areas of the cercal surface, hairs with identical tuning are found at several different and widely separated locations. Furthermore, in some regions of the cerci, filiform hairs with opposite directional tuning are located adjacent to one another. A direct projection of afferents into the ganglion that maintained the nearest-neighbor relationships of the associated hairs on the cercal surface thus would produce a very discontinuous and fragmented image of the sensory world. This is not the case, and this map, therefore cannot be considered to be a simple topographic projectional map.

This cercal afferent array does, however, meet the criteria for a synthetic map. Specifically, the continuous representation of the relevant sensory parameter is synthesized within the nervous 


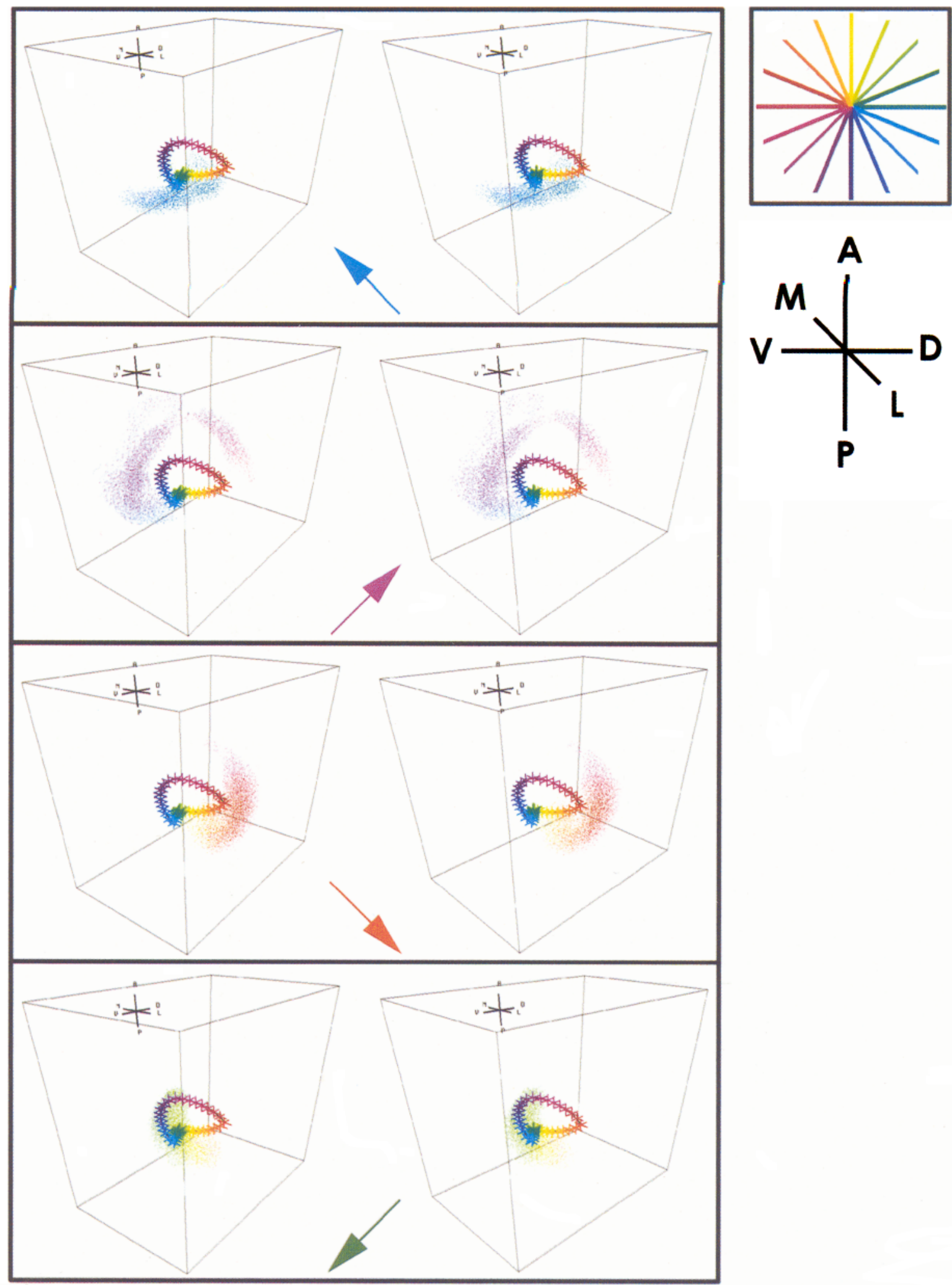

Figure 11. Predicted spatial patterns of excitation within the neural map. The four panels show the spatial excitation patterns in response to stimuli at four orthogonal directions. The stimulus direction with respect to the horizontal plane around the animal's body is indicated with a colored arrow. Each pattern is shown as a stereo pair to emphasize the three-dimensional shape of the excitation pattern. Each pattern is unique and spans a broad region of the neural map. Note that each pattern is composed of several directional regions (indicated by different colors). The colored crosses indicate the centers of mass of 36 different directional regions in the map. Viewing angle is identical to Figure $10, C$ and $D$. 


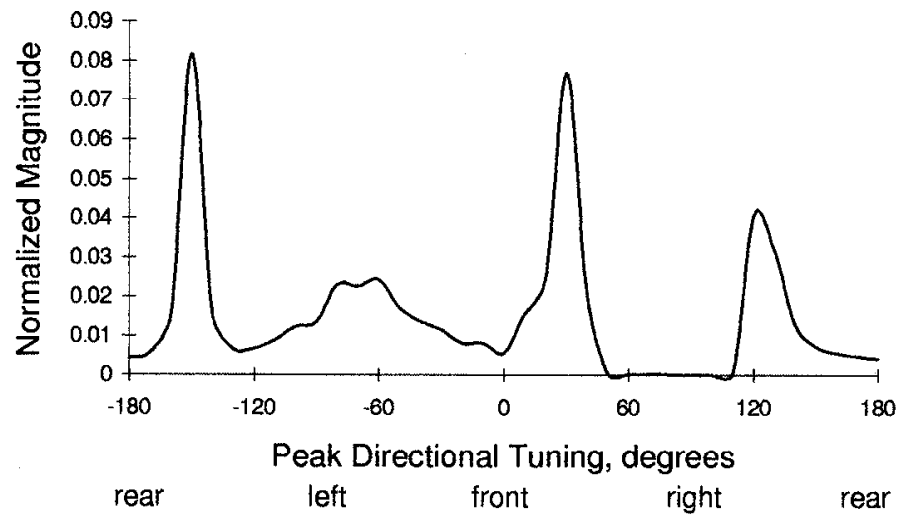

Figure 12. Nonuniform representation of direction within different regions of the neural map. The summed magnitude of all directional vectors with identical peak direction in different regions of the map has been plotted as a function of net directional tuning angle in the map. The spiral of the functional map shown in Figure $10 D$ has been unwound and forms the $x$ axis of the plot. The overall density of points for each peak direction is shown on the $y$ axis. Certain functional locations in the map were greater in magnitude than others. These regions correspond approximately to the peak tuning directions of the largest numbers of afferents in the receptor array.

system via a structural reorganization of the afferent inputs according to aspects of their stimulus/response properties that are independent of the relative locations of the receptors at the periphery. Specifically, a circular, one-dimensional stimulus parameter (i.e., direction with respect to the animal's body) is represented as a three-dimensional spiral of terminal arborizations in the nervous system. It is this anatomical arrangement of the afferent ensemble that sets up the subsequent computations of stimulus direction by the primary interneurons.

How does the representation of stimulus direction within the neural map compare with the representation of direction among the population of afferents that make up the receptor array? The receptor array on each cercus consists primarily of afferents with peak directional tuning clustered around four orthogonal stimulus directions. This nonuniform distribution of afferents forms a continuous map of stimulus direction in the cercal glomerulus. The tuning curves and the axonal arbors of the afferents are broad and overlapping; therefore, the directional tuning associated with any location in the map is derived from the contributions of many afferents, each with a different peak directional tuning.

Although the representation of direction is continuous around the spiral trajectory, the density of afferent terminals was not uniform around the spiral. In Figure 12, the probability density of afferent terminals in the map has been plotted as a function of net directional tuning angle in the map. Effectively, the spiral of the functional map shown in Figure $10 D$ has been unwound and forms the $x$ axis of the plot. There was a much higher density of terminals within certain anatomical locations of the map. The functional tuning of these anatomical locations approximately matches the distribution of peak tuning directions among the population of filiform receptors. Thus, the nonuniform distribution in afferent directional selectivity was preserved in the density distribution of terminals within the map.

\section{Stimuli are represented in spatial patterns of activity}

One general consequence of the ordered representation of sensory parameters in neural maps is that sensory stimuli are repre- sented as specific spatial patterns of activity within the nervous system (Knudsen et al., 1987; Heiligenberg, 1991; Stein and Meredith, 1993). The spatial patterns constitute neural images of the sensory stimuli that must be decoded by a network of postsynaptic interneurons. Based on our global reconstruction of the afferent map, it was possible to predict the steady-state spatial patterns of activity within the ensemble of afferents that would be expected to be caused by several different stimuli (Fig. 11). Each different stimulus activated a broad, three-dimensional region of the map, but each spatial pattern of activation was uniquely correlated with the stimulus. The breadth of the tuning curves of the afferents appeared to be the primary factor responsible for the apparent breadth of these activation patterns. The directional tuning curves of the receptors span the full $360^{\circ}$ of the horizontal plane and are broadly overlapping with one another (Landolfa and Miller, 1995). The afferents are spontaneously active under ambient conditions, and their firing rates are modulated upward or downward as a function of the stimulus direction. Thus, any air current stimulus will cause a change in the global pattern of activation of all terminal varicosities within both cercal glomeruli.

This finding runs counter to the conventional wisdom that any point stimulus (such as a spot of light in the visual field) is represented by a change in activity within a neural map that would be very restricted in spatial extent (Knudsen et al., 1987; Nelson and Bower, 1990; Heiligenberg, 1991; Stein and Meredith, 1993). In the cricket cercal system, each directional stimulus will be represented by a unique three-dimensional pattern of activity that encompasses the entire map. The pattern represents both the increase in activity in some afferents and a decrease in activity in other afferents.

\section{The cercal system and orientation behavior}

The functional organization of the cercal system appears to share many common features with other sensory systems involved in orientation bchaviors. The ncural projection pattern of the afferents represents a map of sensory space in body coordinates that the animal may use to detect approaching predators, mates, or conspecifics (Camhi et al., 1978; Kamper, 1984; Kamper and Dambach, 1985; Heinzel and Dambach, 1987; Bohm and Heidlebach, 1989; Gnatzy and Kamper, 1990; Heidlebach and Dambach, 1995). Although the task of locating stimuli with respect to body coordinates is very similar to that faced by other animals, the precise functional organization of the neural circuits is quite different across species.

First, in the cercal system, there appears to be a uniform representation of all directions in the horizontal plane. This contrasts with visual and auditory maps of space in cats and owls, in which regions of space directly in front of the animal are over-represented via a cortical magnification (Knudsen et al., 1987; Kass, 1991; Stein and Meredith, 1993). Second, the cercal system is highly redundant. Afferents with similar directional tuning are found at multiple locations on each cercus, and afferents from each cercus form a complete map of sensory space on each side of the ganglion. Thus, the cercal system should be relatively insensitive to the loss of several receptors, and even to the loss of an entire cercus. Unlike the retina, losing receptors from a localized region of the cercal epithelium would not create a blind spot in the representation of stimulus space.

The cercal system may be the first known example of a synthetic map created at the level of primary afferents. It differs from other synthetic maps in that the computation of direction is not achieved by comparing activity levels between two sensory organs, 
such as between two ears (Carr and Konishi, 1990) or between different body locations, as in electric fish (Carr et al., 1986a,b; Heiligenberg, 1987). The combined activity from both cerci act in concert to create a unique global pattern of activity in the entire map that represents the location of a stimulus with respect to body coordinates.

The functional representation of stimulus direction in the cercal system is similar to the map of visual space in the deep layers of the superior colliculus in cats (Meredith and Stein, 1990). The functional organization that emerges from these ensembles of neurons with large, overlapping receptive fields is large blocks of neural tissue that view the same region of visual space. Thus, in both systems a relatively coarse grained map of sensory space has been implemented, not for the precise discrimination of objects but for the location of sensory stimuli with respect to body coordinates.

Unlike the more superficial layers of the superior colliculus, the computations that take place within the neural map of stimulus direction appear to be limited to a single sensory modality: the detection of air currents. This sensory information projects to the thorax and brain, where it is integrated with other sensory modalities: vision, auditory input, and tactile input to the body and antennae. Groups of thoracic interneurons in the cockroach cercal system are multimodal and respond to specific combinations of sensory stimuli (Ritzmann et al., 1991; Pollak et al., 1995). It is not known, however, whether additional maps of auditory and visual space exist in the thoracic ganglia; nor is it known whether any such maps are aligned with somatotopic maps of the body (Johnson and Murphey, 1985) or with a map of stimulus direction. Rehavioral experiments suggest that tactile inputs to the antennae can alter the response to wind puffs directed at the cerci which, in turn, suggests that the evaluation of body position in the environment is a function of the integration of inputs from many sensory modalities (Stierle et al., 1994; Watson and Ritzmann, 1994). Thus, in many ways the cercal system may be equivalent in an operational sense to the visual or auditory system of the animal-one that functions as a constant monitor of the local environment.

\section{REFERENCES}

Bacon J, Murphey RK (1984) Receptive fields of cricket giant interneurons are related to their dendritic structure. J Physiol (Lond) 352:601-623.

Bohm H, Heidlebach J (1989) Processing of wing flick signals in cercusto-giant interneurons of the African cave cricket. In: Neural mechanisms of behavior (Erber J, Menzel R, Pfluger HJ, Todt D, eds). Stuttgart: Georg Thieme.

Carr CE, Konishi M (1990) A circuit for detection of interaural time differences in the brain stem of the barn owl. J Neurosci 10:3227-3246.

Can CE, Heiligenberg W, Ruse GJ (1986a) A time-comparison circuit in the electric fish midbrain. I. Behavior and physiology. J Neurosci 6:107-119.

Carr CE, Maler L, Taylor B (1986b) A time-comparison circuit in the electric fish midbrain. II. Functional morphology. J Neurosci 6:1372-1383.

Camhi JM, Tom W, Volman S (1978) The escape behavior of the cockroach Periplaneta americana. II. Detection of natural predators by air displacement. J Comp Physiol 128:203-212.

Edwards JS, Palka J (1974) The cerci and abdominal giant fibres of the house cricket Acheta domesticus. I. Anatomy and physiology of normal adults. Proc R Soc Lond [Biol] 185:83-103.

Efron B (1982) The jack knife, the bootstrap and other resampling plans. Philadelphia: Society for Industrial and Applied Mathematics.
Gnatzy W, Kamper G (1990) Digger wasp against crickets. II. An airborne signal produced by a running predator. J Comp Physiol $167: 551-556$.

Gnatzy W, Tautz J (1980) Ultrastructure and mechanical properties of an insect mechanoreceptor: stimulus-transmitting structures and sensory apparatus of the cercal filiform hairs of Gryllus. Cell Tissue Res 213:441-463.

Heidlebach J, Dambach M (1995) Wing flick behaviour in the nonstridulating African cave cricket Phaeophilacris spectrum. Proceedings of the 4th International Congress of Neuroethology.

Heiligenberg W (1987) Central processing of sensory information in electric fish. J Comp Physiol 161:621-631.

Heiligenberg W (1991) The neural basis of behavior: a neuroethological view. Annu Rev Neurosci 14:247-267.

Heinzel HG, Dambach M (1987) Travelling air vortex rings as potential communication signals in a cricket. J Comp Physiol 160:79-88.

Jacobs GA, Miller JP (1985) Functional properties of individual neuronal branches isolated in situ by laser photoinactivation. Science 228:344-346.

Jacobs GA, Murphey RK (1987) Segmental origins of the cricket giant interneuron system. J Comp Neurol 265:145-147.

Jacobs GA, Nevin R (1991) Anatomical relationships between sensory afferent arborizations in the cricket cercal system. Anat Rec 231:563-572.

Jacobs GA, Miller JP, Murphey RK (1986) Cellular mechanisms underlying directional sensitivity of an identified sensory interneuron. J Neurosci 6:2298-2311.

Johnson SE, Murphey RK (1985) The afferent projection of mesothoracic bristle hairs in the cricket Acheta domesticus. J Comp Physiol $156: 369-379$.

Kaas JH (1991) Plasticity of sensory and motor maps in adult mammals. Annu Rev Neurosci 14:137-167.

Kamper G (1984) Abdominal ascending interneuron in crickets: responses to sound in the $30-\mathrm{Hz}$ calling-song frequency. J Comp Physiol 155:507-520.

Kamper G, Dambach M (1985) Low-frequency airborne vibrations generated by crickets during singing and aggression. J Insect Physiol 31:925-929.

Kamper G, Kleindienst H (1990) Oscillation of cricket sensory hairs in a low-frequency sound field. J Comp Physiol 167:193-200.

Knudsen EI, du Lac S, Esterley SD (1987) Computational maps in the Brain. Annu Rev Neurosci 10:41-65.

Konishi M (1991) Deciphering the brain's codes. J Neural Comput 3:1-18.

Landolfa MA, Jacobs GA (1995) Direction sensitivity of the filiform hair population of the cricket cercal system. J Comp Physiol [A] 177:759-766.

Landolfa MA, Miller JP (1995) Stimulus-response properties of cricket cercal filiform receptors. J Comp Physiol 177:747-757.

Mendenhall B, Murphey RK (1974) The morphology of cricket giant interneurons. J Neurobiol 5:565-580.

Meredith MA, Stein BE (1990) The visuotopic component of the multisensory map in the deep laminae of the cat superior colliculus. $J$ Neurosci 10:3727-3742.

Miller JP, Jacobs GA, Theunissen FE (1991) Representation of sensory information in the cricket cercal sensory system. I. Response properties of the primary interneurons. II Neurophysiol 66:1680-1689.

Murphey RK (1981) The structure and development of a somatotopic map in crickets: the cercal afferent projection. Dev Biol 88:236-246.

Murphey RK, Lemere CA (1984) Competition controls the growth of an identified axonal arborization. Science 224:1352-1355.

Murphey RK, Jacklet A, Schuster L (1980) A topographic map of sensory cell terminal arborizations in the cricket CNS: correlation with birthday and position in a sensory array. J Comp Neurol 191:53-64.

Nelson ME, Bower JM (1990) Brain maps and parallel computers. Trends Neurosci 13:403-408.

Palka J, Levine RB, Schubiger M (1977) The cercus-to-giant interneuron system of crickets. I. Some attributes of the sensory cells. J Comp Physiol 119:267-283.

Pollack AJ, Ritzmann RE, Watson JT (1995) Dual pathways for tactile sensory information to thoracic interneurons in the cockroach. J Neurobiol 26:33-46.

Ritzmann RE, Pollack AJ, Hudson SE, Hyvonen A (1991) Convergence of multi-modal sensory signals at thoracic interneurons of the escape system of the cockroach, Periplaneta americana. Brain Res $563: 175-183$. 
Shimozawa T, Kanou M (1984a) Varieties of filiform hairs: range fractionation by sensory afferents and cercal interneurons of a cricket. $J$ Comp Physiol 155:485-493.

Shimozawa T, Kanou M (1984b) The aerodynamics and sensory physiology of range fractionation in the cercal filiform sensilla of the cricket Gryllus bimaculatus. J Comp Physiol 155:495-505.

Stein BE, Meredith MA (1993) The merging of the senses. Cambridge, MIT.

Stierle IE, Getman M, Comer CM (1994) Multisensory control of escape in the cockroach Periplaneta americana. I. Initial evidence from patterns of wind-evoked behavior. J Comp Physiol 174:1-11.

Theunissen FE, Miller JP (1991) Representation of sensory information in the cricket cercal sensory system. II. Information theoretic calculation of system accuracy and optimal tuning-curve widths of four primary interneurons. J Neurophysiol 66:1690-1703.
Tobias M, Murphey RK (1979) The response of cercal receptors and identified interneurons in the cricket (Acheta domesticus) to airstreams. J Comp Physiol 179:51-59.

Troyer TW, Levin JE, Jacobs GA (1994) Construction and analysis of a database representing a neural map. Microsc Res Tech 29:329-343.

Walthall WW, Murphey RK (1986) Positional information, compartments, and the cercal sensory system of crickets. Dev Biol 113:182-200.

Watson JT, Ritzmann RE (1994) The escape response versus the quiescent response of the American cockroach: behavioural choice mediated by physiological state. J Neurobiol 48:476-478.

Westin J (1979) Responses to wind recorded from the cercal nerve of the cockroach Periplaneta americana: response properties of single sensory neurons. J Comp Physiol 133:97-102. 